\title{
Retinal Image Classification using a Histogram Based Approach
}

\author{
Mohd Hanafi Ahmad Hijazi, Frans Coenen and Yalin Zheng
}

\begin{abstract}
An approach to classifying retinal images using a histogram based representation is described. More specifically, a two stage Case Based Reasoning (CBR) approach is proposed, to be applied to histogram represented retina images to identify Age-related Macular Degeneration (AMD). To measure the similarity between histograms, a time series analysis technique, Dynamic Time Warping (DTW), is employed. The advocated approach utilises two "case bases" for the classification process. The first case base consists of green and saturation histograms with retinal blood vessels removed. The second case base comprises the same histograms, but with the Optic Disc (OD) removed as well. The reported experiments demonstrate that the proposed two stage classification process outperforms the single stage classification process with respect to a number of evaluation metrics: specificity, sensitivity and accuracy.
\end{abstract}

\section{INTRODUCTION}

In this paper a mechanism for classifying retina images to identify Age related Macular Degeneration (AMD), the most common cause of reduced vision in people over sixty years of age, is described. The mechanism uses a histogram based approach to represent retina images. The classification, in turn, is founded on a Case Based Reasoning (CBR) mechanism coupled with a Dynamic Time Warping (DTW) technique. A given, pre-labelled, training set is processed so that each retina image is represented by a collection of histograms, extracted with respect to the RGB (Red, Green and Blue) and HSI (Hue, Saturation and Intensity) colour models. The number of histograms was reduced by selecting only the green channel and saturation component histograms. The green channel was selected as it has been show to offer the best discriminatory power between the main retinal anatomy (blood vessels, fovea and optic disc) and the retinal background [1]. Saturation was chosen because it has also been shown to give a good performance in identifying AMD images, as established by a series of experiments conducted by the authors [2]. These histograms are conceptualised as time series, i.e. a set of curves. The curves are stored in two Case Bases $(C B s)$. The first case base $(C B 1)$ comprises green and saturation histograms generated from retina images that have been enhanced and had blood vessel pixels removed. The second case base $(C B 2)$ comprises green and saturation histograms from retina images that have been further processed by removal of the Optic Disc (OD) pixels. Removed pixels are replaced with "null" pixels.

Mohd Hanafi Ahmad Hijazi is with the Department of Computer Sciences, The University of Liverpool, Liverpool, L69 3BX (phone: +44 151 795 4294; email: m.ahmad-hijazi@ liverpool.ac.uk).

Frans Coenen is with the Department of Computer Sciences, The University of Liverpool, Liverpool, L69 3BX (phone: +44 151795 4253; email: coenen@liverpool.ac.uk).

Yalin Zhang is with the Unit of Ophthalmology, School of Clinical Sciences, The University of Liverpool, Liverpool, L69 3GA (phone: +55 151706 4083; email: yzheng @ liverpool.ac.uk).
The classification is a two stage process. During the first stage "unseen data" is classified by finding the most similar match in $C B 1$. (The unseen data is usually presented as a collection of retina images, rather than a single image.) The similarity matching is conducted using the DTW technique. For those unseen images that can be classified with a certain degree of certainty the process stops. For the remaining images that cannot be classified with any certainty the process is repeated with $C B 2$. $C B 2$ is not used immediately because the optic disc removal process, in some cases, has the effect of removing significant pixels. The results presented in this paper demonstrate that this two pass approach produces better results than when using a one pass approach (using either $C B 1$ or $C B 2$ in isolation).

The focus of the work described is the detection of AMD. AMD is a condition where the macula, a small area located at the very centre of the retina, as shown in Figure 1(a), degenerates with age [3]. The first clinical indicator of AMD is drusen. Drusen are yellowish sub-retinal deposits that are often difficult to identify against the predominantly orange retina background. The severity of AMD is categorised as being either: early, intermediate, advanced non-neovascular, or advanced neovascular [3]. Each category is characterised by the existence of various sizes and shapes of drusen as well as pigment abnormality. An example of a retina image that features drusen is given in Figure 1(b) (indicated by the white arrow). The drusen itself are categorised as hard and soft drusen. Hard drusen have a well defined border, while soft drusen have boundaries that often blend into the background. In some cases the drusen "blends" with the optic disc. The identification of drusen, especially soft drusen, is thus not a straightforward process [4], [5].

Given the increasing incidence of AMD, attempts have been made in many countries to establish screening programmes. However, the manual processing of retinal images is labour intensive. The accuracy of the screening is also subject to the graders observation [6]. The automation, or partial automation, of the process is therefore desirable although the complexity of drusen detection hampers such automated screening, especially in the case of early stage AMD when the presence of drusen is often difficult to detect. The technique prescribed in this paper is intended to provide new technical support for the screening process.

The rest of this paper is organised as follows. Section II presents some necessary background to support the work described. Section III describes the classification process in more detail. Section IV describes the image preprocessing techniques applied to the input data to create $C B 1$ and $C B 2$. The specific DTW approach developed is detailed in Section $\mathrm{V}$. An evaluation of the proposed approach is then given 


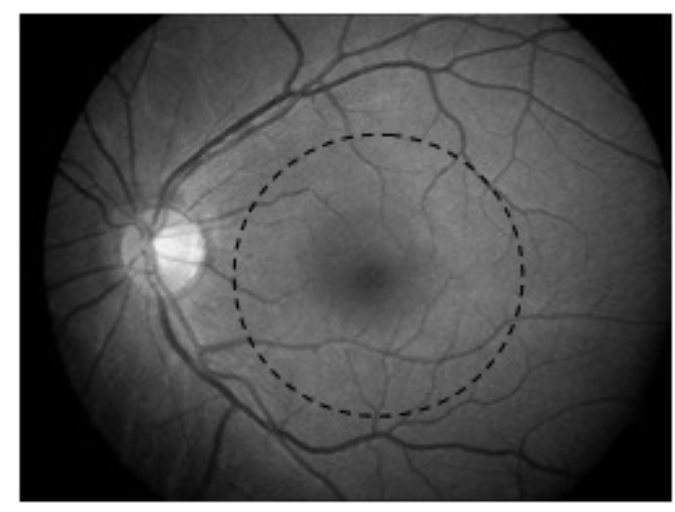

(a)

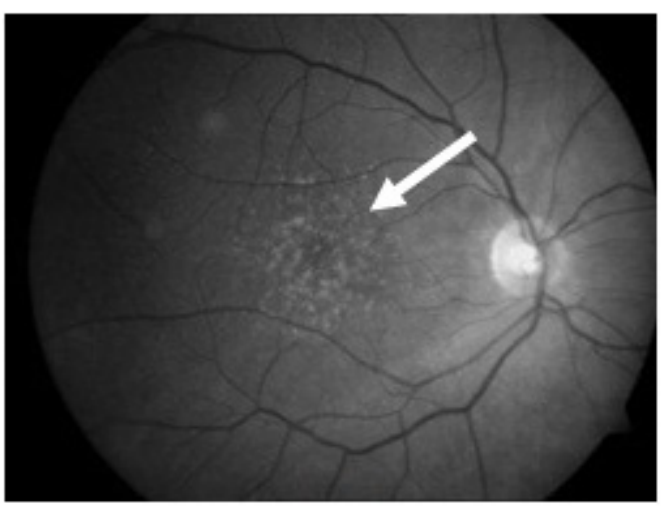

(b)

Fig. 1. (a) Normal and (b) AMD retinal images in greyscale. The circled area indicates the Macula. The white arrow indicates drusen

in Section VI, followed by some conclusions presented in Section VII.

\section{Previous Work}

There are a number of references to image classification and retrieval using histogram comparison techniques that have been reported in the literature, examples include [7], [8], [9]. The fundamental idea is to encode the colour information in an image as a feature vector and then represent the vectors as histograms. The distinguishing element of these methods is the similarity measures utilised to compare histograms; examples include Earth Mover's Distance (EMD) [10], Manhattan distance $\left(L_{1}\right)$ and Euclidean distance $\left(L_{2}\right)$ [11].

The above histogram comparison techniques assume that the collection of histograms each have an equal number of sample points, time series analysis techniques are directed at more varied categories of sequential data representation. For example Al-Aghbari [7] proposed a time series analysis approach to address the histogram based image classification problem, which has similarities with the work reported in this paper. Using a time series data representation, called Symbolic Aggregate approXimation (SAX), and the Knearest neighbour technique for classification, the results in [7] demonstrated a promising approach to the use of time series analysis for image mining. The distinction between
Al-Aghbari's approach and that presented in this paper is in terms of the time series data representation and the classifier used. High data dimensionality is not an issue with respect to colour histograms as the length of the time series data is bounded (typically to 256 sample points). The approach described here can thus use a "normal" histogram representation. A DTW technique has been proposed in this paper to compare histograms.

Histograms can be generated from images in a number ways. The most straight forward is to to generate sets of histograms from the three colour channels used to represent images (Red-Green-Blue or RGB). Another option is to use Hue-Saturation-Intensity (HSI) values. Experiments reported in [4], [12] and [1], have shown that the most appropriate colour channel for object identification (including drusen) in retina images is the Green colour channel. Since the classification of AMD images is entirely based on the retinal colour distribution which will be severely affected by the quality of the captured images, this paper proposes the use of saturation histograms for additional coroboration. Saturation was selected, as opposed to hue or intensity, as it also demonstrated good performance in identifying AMD in retinal images, this was established through a series of experiment conducted by the authors and reported in [2].

CBR [13] is an established AI technique founded on the observation that humans solve new problems by referring back to similar problems they have solved in the past, i.e. a Case Base of past cases. Given a new case a CBR system will find the most similar past cases (or cases) in its Case Base and present the solution(s) to the identified case(s) as solution(s) to the new case. One of the most significant issues in $\mathrm{CBR}$ is the nature of the similarity checking mechanisms used to identify similar cases in the Case Base. In the work described here we use a DTW technique.

DTW [14] is a time series analysis technique [15] for comparing two curves and generating a similarity measure. It uses a dynamic programming approach to align two time series and then generates a "warping path" that maps (aligns) the two sequences onto each other. Further details on the use of DTW, in the context of this paper, are given in Section V.

\section{Retina Image Classification Process}

An overview of the retina image classification process, to identify AMD, is presented in this section. The process can be conceptualised as a two stage process: (i) case base generation, and (ii) classification of unseen data.

The case base generation process is presented in Figure 2. The process commences with a training set of pre-labelled images $(D B)$. Due to uneven illumination, the colour retinal images were first normalised using the histogram specification technique [16]. The images were then further enhanced to emphasise the contrast within the images as well as the visibility of the main retinal anatomy edges. The retinal blood vessels and OD, which are deemed to be "noise", may be removed by replacing them with null values (this process is described in further detail in Subsection IV-B). The preprocessed images are then converted into histograms and 
the green and saturation histograms retained (for reasons described in Section II above. These are then converted into time series (curves) and stored in the first case base $(C B 1)$. The images are then processed further and the optic disc removed and replaced with null pixels. It should be noted that removal of the optic disc is not $100 \%$ accurate, especially in images of severely damaged retinas. However, the classification of images with severely damaged retinas is relatively straightforward. Thus the expectation is that such images will be classified solely with reference to $C B 1$ and will not need to reference the second case base $(C B 2)$; the ckassification process is described in further detail below. Once the second image pre-processing stage is complete, a second set of histograms are produced (again green and saturation only) and $C B 2$ generated.

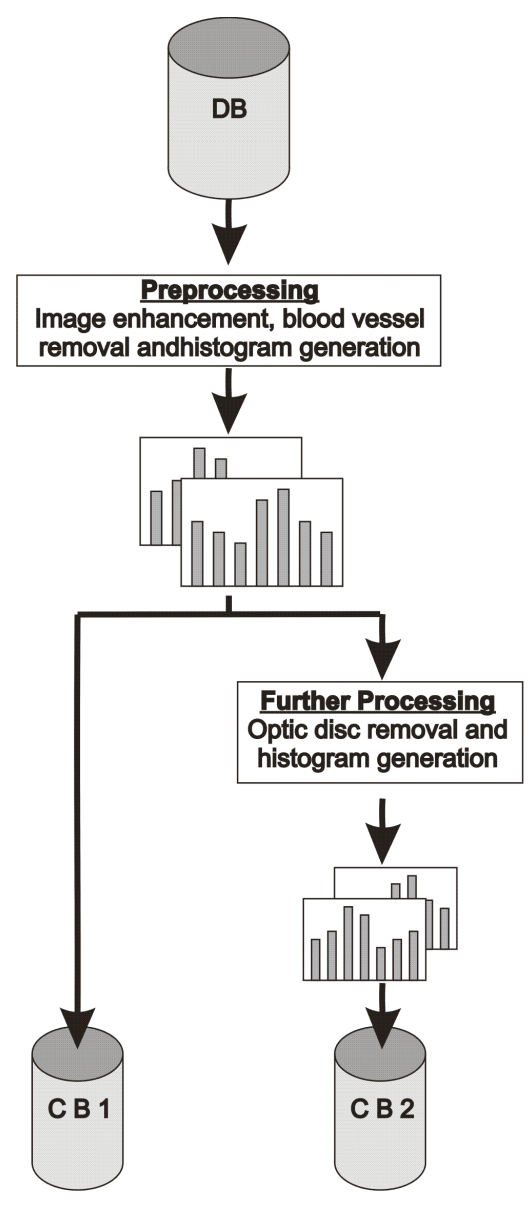

Fig. 2. Case base generation

Once both $C B 1$ and $C B 2$ are in place the classification of unseen data can commence. The process (Figure 3), like the case base generation process described above, is a two stage process. The process commences with a collection of one or more retina images to be labeled as AMD or not AMD. These are initially pre-processed in the same way as before to enhance the images and remove blood vessel data. These are then passed to a "Case Based Reasoner" which interacts with $C B 1$ to find the most similar curves in the case base. Comparison is undertaken using the proposed DTW technique (see Section V). The curves for the current retina image are compared with those in the case base and a similarity measure $(\sigma)$ produced for each comparison. If:

1) There are no values of $\sigma$ above a similarity threshold; or

2) More than one curve, with different associated class labels, have a similar $\sigma$,

we go onto stage two. If there is a clear "winner" the label for the most similar curve in $C B 1$ is selected and the classification process, with respect to the current image, stops. Otherwise we go onto stage two. The current image is processed further and the optic disc removed, and then passed to the second "Case Based Reasoner" which interacts with $C B 2$. Using the same DTW technique as before, the label associated with the most similar curve in the case base is selected as the class for the current retina image.

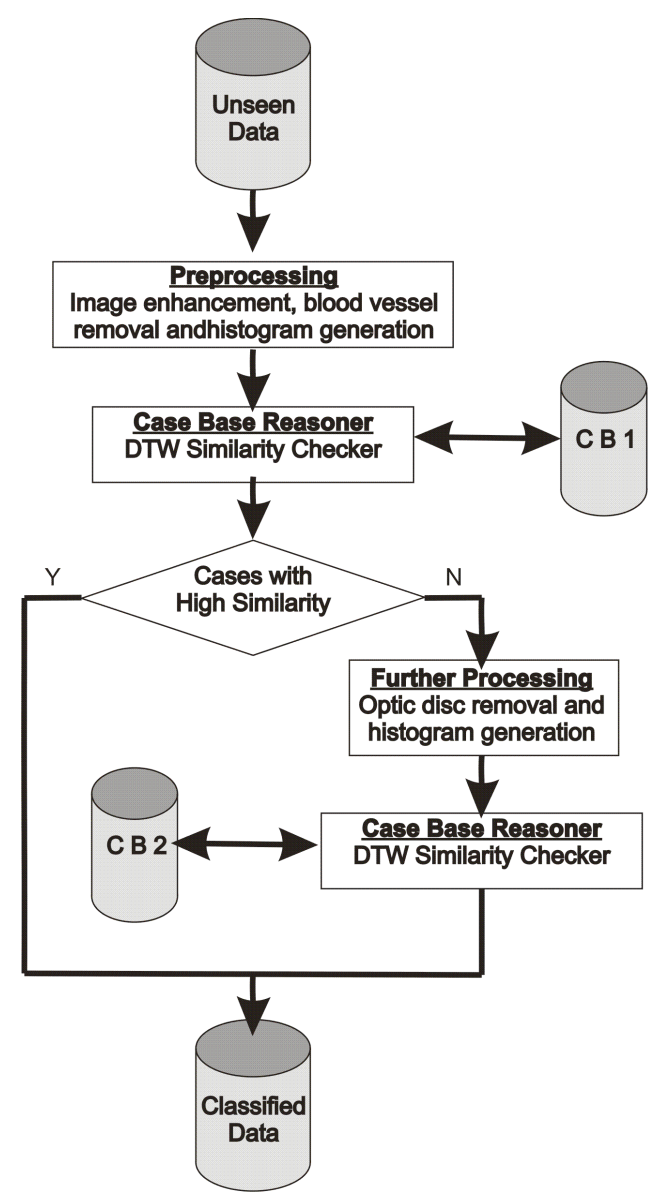

Fig. 3. Classification process applied to unseen data

\section{IMAGE PREPROCESSING}

This section describes the two principle image preprocesses used during the processing of images as described above: namely image enhancement (Sub-section IV-A) and noise elimination (Sub-section IV-B). 


\section{A. Image Enhancement}

The quality of the retinal images, which are acquired with a digital fundus camera, is heavily effected by factors that are difficult to control. The most common problem is nonuniform illumination ([17], [18]), where the luminosity and contrast variability are unevenly distributed across the image. Figure 4(a) shows that the illumination exposure is lower towards the perimeter of the retina but higher in the middle. This may be caused by number of conditions, such as the subject's eye movement and presence of other diseases that may block the light reaching the retina (such as cataracts), and retinal colour variations. Thus, colour normalisation and contrast enhancement are necessary before any diagnosis can take place.

In this paper, a histogram specification approach was applied to normalise the retinal images colour. This approach is used to normalise each image to a desired property, such as its frequency histogram, to a reference image distribution ([16], [18]). First, a reference image that displays the best colour distribution and contrast, is carefully selected by a trained clinician. The histograms of the reference image are then generated. Next, the histograms of the other images are extracted and each of these histograms is tuned to match the reference image histograms. This approach has been proven to produced colour normalised retinal images [18]. Figure 4(b) shows the colour normalised image of Figure 4(a).

Once the colour is normalised, contrast enhancement is applied to make objects in the retina more visible. One common contrast enhancement technique, in the context of histograms, is histogram equalisation (HE). HE spreads out the most frequent intensity values to produced a better distributed histogram. Through this transformation, the contrast of an image is improved globally; unfortunately, the enhancement results in some bright parts of the image being further enhanced to the extent that they are "over exposed" and consequently edges become less distinct. To overcome this problem, Contrast Limited Adaptive Histogram Equalisation (CLAHE) [19] was adopted. CLAHE computes several histograms that correspond to different sections of an image and equalise each histograms independently. The authors have conducted empirical experiments with a variety of histogram based contrast enhancement techniques (not reported here) from which it was concluded that CLAHE gave the best performance. The retina edges and the OD boundary in Figure 4(c), to which CLAHE enhancement has been applied, is clearly more visible and identifiable.

\section{B. Noise Elimination}

The identification of fundamental retinal anatomies, such as retinal vessels and the optic disc, is necessary before any complex tasks of identifying pathological entities can be undertaken ([4], [5], [20]). Knowledge of the location of these structures is often used to provide reference points to which the detection of eye related diseases can be related. However, the process is not straightforward because their appearances on the fundus images are affected by illumination variations

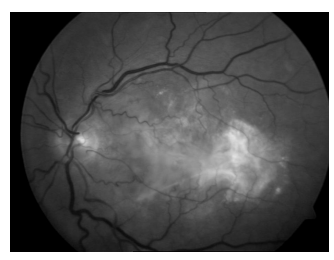

(a)

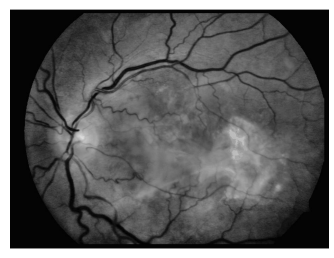

(c)

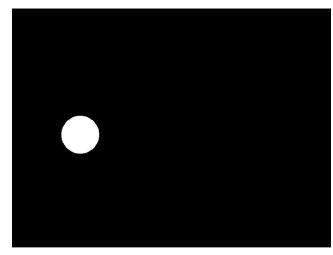

(e)

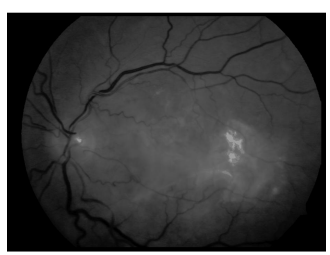

(b)

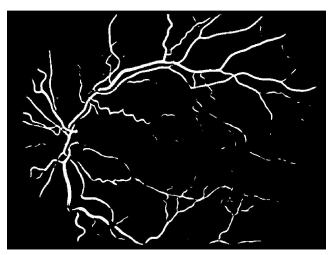

(d)

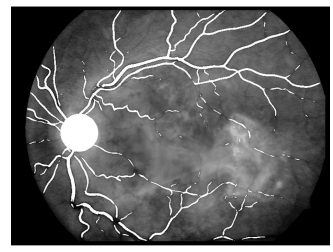

(f)
Fig. 4. Retinal green channel image: (a) original image, (b) colour normalised, (c) contrast enhanced, (d) retinal vessels binary image, (e) Optic Disc (OD) segmented binary image and (f) retinal vessels and OD removed

as well as the severity of the retinal disease. With respect to AMD classification the optic disc in particular is sometimes identified as drusen because they share similar features (both are yellowish in colour, and brighter when compared to the background of the retina). The retinal vessels, on the other hand, may effect the colour distribution of an image, which in our case is not desirable. It is has been established, by the authors, that by focusing only on the colour distribution of the retinal background and the sypmtoms of AMD (if any), a better classifier will be produced.

To segment the retinal vessels, a common matched filters algorithm as proposed by Chaudhuri et al. [1] was used. This approach has been proved to achieved a good performance if applied to a well enhanced image [12]. For the segmentation of retinal vessels, a kernel of size $15 \times 15$ is generated and rotated into twelve different orientations $\left(0^{\circ}\right.$ to $\left.165^{\circ}\right)$. These kernels are applied to each pixel where only the maximum response is kept for each pixel. To obtain a retinal vessels binary image (Figure 4(d)), the response image is then thresholded using the Otsu's threshold algorithm [21]. The images with enhancement and blood vessels removed are then used to create $C B 1$

An approach where by the $2 D$ retinal image is projected onto two $1 D$ signals (representing the horizontal and vertical axis of the retinal image), similar to that proposed in [22] and [5], was adopted to identify the location of the OD. The number of horizontal and vertical edges together with the 
intensity value of a retinal image were used as features to localised the OD. Instead of using the original green channel image, as proposed in [22], the authors propose the uses of the retinal vessels image and the enhanced green channel image to generate the (vertical and horizontal) $1 D$ signals. To identify the horizontal location of the OD, a sliding window of double the thickness of the identified main retinal vessel, $\varpi$, and the image height was used to scan the edge maps image from left to right and project the image features of each window onto the horizontal axis $1 D$ signal. The horizontal location of the OD was identified by the maximum peak of the $1 D$ signal. A vertically sliding window, with the size of the OD diameter, $\delta$, was then used to scan the image from top to bottom in-line with the OD's identified horizontal location. A vertical axis $1 D$ signal was thus produced and the vertical location of the OD identified by the maximum peak of the vertical axis $1 D$ signal. The OD is then segmented using a template with a prescribed radius, $\rho$, whose value is dependent on the image size. In this paper, the values of $\varpi, \delta$ and $\rho$ have been predetermined through experiments ( $\varpi=13$ pixels, $\delta=131$ pixels and $\rho=45$ pixels). Figure 4(e) shows the OD segmented binary image. The identified retinal vessels and OD location can then be removed from the retinal image (Figure 4(f)). The OD removal process is applied to create $C B 2$.

\section{DYNAMIC TIME WARPING}

As noted in Section II DTW is a well established time series analysis technique. The particular variation of DTW developed by the authors for comparing retinal histograms is described in this section.

The application of DTW to time series analysis ([23], [24]) is as follows. To map two time series $T$ and $S$, of length $n$ and $m$ respectively, where $T=t_{1}, t_{2}, \ldots, t_{n}$ and $S=s_{1}, s_{2}, \ldots s_{m}$, a $n$-by- $m$ matrix will be formed, where the $\left(i^{t h}, j^{t h}\right)$ grid points corresponds to the alignment or distance between any two points $t_{i}$ and $s_{j}$. The warping path, $W$, is then the set of matrix elements that identifies a mapping between $T$ and $S$, defined as $W=w_{1}, w_{2}, \ldots w_{K}$, where $\max (m, n) \leq K<m+n-1$. The distance $d\left(t_{i}, s_{j}\right)$ between two points $t_{i}$ and $s_{j}$ is used to identify potential warping paths. There are many distance measure that may be used, the most common is the Euclidean distance, and this is the measure used in this paper. Thus,

$$
d\left(t_{i}, s_{j}\right)=w_{k}=\left(t_{i}-s_{j}\right)^{2}
$$

The minimal warping path is selected by calculating the minimum cumulated distance between $T$ and $S$ as:

$$
\operatorname{DTW}(T, S)=\min \left[\sqrt{\sum_{k=1}^{K} w_{k}}\right]
$$

In this paper, the green channel and saturation component histograms are extracted from the colour normalised and contrast enhanced (as described in Subsection IV-A) retinal images, represented in the form of histograms. The retinal blood vessel pixels (generated as described in Subsection IV-B) are subsequently removed by subtracting the vessels intensity value from the generated histograms. These histograms formed the first "case base" $C B 1$ comprising: green, $G=g_{0}, g_{1}, \ldots, g_{i}$, and saturation, $S=s_{0}, s_{1}, \ldots, s_{I}$, histograms (where $I$ is the number of case base images and $G, S \in C B 1$ ). Next, the optic disc pixels are removed as well and formed the second "case base" $C B 2$. New "unseen" images to be classified formed a set of $J$ "new cases" $\bar{C}$, comprising: green, $\bar{G}=\bar{g}_{0}, \bar{g}_{1}, \ldots, \bar{g}_{J}$, and saturation, $\bar{S}=\bar{s}_{0}, \bar{s}_{1}, \ldots, \bar{s}_{j}$, histograms (where $J$ is the number of unseen images and $\bar{G}, \bar{S} \in \bar{C}$ ). In the classification stage, each curve in $\bar{G}$ is compared with the content of $G$ using DTW. A list of the $n$ most similar $g \in G(n=5$ in this paper), $\operatorname{sim}(\bar{g})$ is produced for each $\bar{g}$ :

$$
\begin{gathered}
\operatorname{sim}\left(\bar{g}_{j}\right)=\left\{\left(g_{0}, \delta_{0}^{j}\right), \ldots,\left(g_{n}, \delta_{n}^{j}\right)\right\} \\
\delta_{n}^{j}=D T W\left(\bar{g}_{j}, g_{i}\right)
\end{gathered}
$$

$\operatorname{DTW}(\bar{g}, g)$ is the minimal warping path or distance of the green channel "new case" histogram, $\bar{g} \in \bar{G}$ and its most similar green channel "case base" histogram, $g \in G$ (equation 2), $0 \leq i<I$ and $0 \leq j<J$. Next, a similar distance measuring process is applied to $\bar{S}$ and $S$ only on $n$ saturation histograms, $s \in S$, which are deemed as most similar to the unseen image according to $\operatorname{sim}(\bar{g})$. This step will produce a list of saturation curve distances, $\operatorname{sim}(\bar{s})$, for each $\bar{s} \in \bar{S}$, of length $n$ :

$$
\begin{gathered}
\operatorname{sim}\left(\bar{s}_{j}\right)=\left\{\left(s_{0}, \bar{\delta}_{0}^{j}\right), \ldots,\left(s_{n}, \bar{\delta}_{n}^{j}\right)\right\} \\
\bar{\delta}_{m}^{j}=\operatorname{DTW}\left(\bar{s}_{j}, s_{i}\right)
\end{gathered}
$$

Finally, the final list of $n$ most similar cases, $c b 1 \in C B 1$ for each $\bar{c} \in \bar{C}$ is generated as follows:

$$
\begin{gathered}
\operatorname{sim}\left(\bar{c}_{j}\right)=\left\{\left(c b 1_{0}, \mu_{0}\right), \ldots,\left(c b 1_{n}, \mu_{n}\right)\right\} \\
\mu_{n}=\frac{1}{2}\left(\delta_{n}^{j}+\bar{\delta}_{n}^{j}\right)
\end{gathered}
$$

If at least two of $c b 1 \in C B 1$ of $\operatorname{sim}(\bar{c})$ have a similar $\mu$, the distance measure processes (equation 1 to equation 8 ) will be applied to $C B 2$. Each "new case", $\bar{c} \in \bar{C}$ will then be classified as belonging to the same class of its most similar histogram in the second "case base", $c b 2 \in C B 2$. Otherwise, by default, $\bar{c} \in \bar{C}$ is classified according to the results of $c b 1 \in C B 1$.

\section{EVALUATION}

To evaluate the proposed retina image classification approach a data set comprising 144 images was used ${ }^{1} .86$ of the image were known to feature AMD, and the remaining 56 were "normal" control images. The images were collected as part of the ARIA project ${ }^{2}$. A Ten-fold Cross Validation

\footnotetext{
${ }^{1}$ In the context of data mining this is considered to be a relatively small data set, however real retina image data to be used for research purposes is difficult to acquire for reasons of "ethical approval".

${ }^{2}$ http://www.eyecharity.com/aria_online/
} 
(TCV) approach was adopted with respect to all the experiments. To illustrate the advantage obtained by the proposed process five sets of experiments were conducted: the first two were using the original image histograms, $I_{o r}$, and enhanced image histograms, $I_{e n}$, each, one using $C B 1$ only, one using $C B 2$ only, and one using the method proposed in this paper (using both $C B 1$ and $C B 2$ ). Three evaluation metrics were used: Specificity, Sensitivity and Accuracy. Sensitivity will measure the effectiveness of the classifier to identify true positives (AMD images), while specificity is used to measure the performance of the classifier in identifying the normal control images (true negatives). Accuracy is used to measure the overall performance of the classifier in term of classifying retinal images correctly according to their class (AMD or normal).

The results of the first two sets of experiments are presented in Table I. The results show the feasibility of using retinal image colour histograms (green and saturation in this paper) in AMD image classification, and a more distinguished class labels (AMD and normal) can be obtained through image enhancement. This is clearly indicated by an average improvement of $2 \%$ in all evaluation metrics used.

TABLE I

EXPERIMENTAL RESULTS GENERATED USING TCV: (I) ORIGINAL GREEN AND SATURATION HISTOGRAMS, $I_{o r}$, AND (II) ENHANCED GREEN AND SATURATION HISTOGRAMS, $I_{e n}$

\begin{tabular}{|l||c|c||c|c||c|c|}
\hline \multicolumn{1}{|l||}{\multirow{2}{*}{$\begin{array}{c}\text { TCV } \\
\text { run }\end{array}$}} & \multicolumn{2}{c||}{ Specificity (\%) } & \multicolumn{2}{c||}{ Sensitivity (\%) } & \multicolumn{2}{c|}{ Accuracy (\%) } \\
\cline { 2 - 7 } & $I_{o r}$ & $I_{e n}$ & $I_{o r}$ & $I_{e n}$ & $I_{o r}$ & $I_{e n}$ \\
\hline 1 & 80 & 40 & 78 & 89 & 79 & 71 \\
2 & 50 & 67 & 88 & 75 & 71 & 71 \\
3 & 67 & 67 & 67 & 67 & 67 & 67 \\
4 & 50 & 67 & 67 & 89 & 60 & 80 \\
5 & 50 & 67 & 100 & 89 & 80 & 80 \\
6 & 67 & 50 & 67 & 100 & 67 & 80 \\
7 & 67 & 33 & 100 & 88 & 86 & 64 \\
8 & 40 & 60 & 67 & 50 & 57 & 64 \\
9 & 33 & 50 & 100 & 75 & 71 & 64 \\
10 & 50 & 67 & 50 & 75 & 50 & 71 \\
\hline Ave. & 55 & 57 & 78 & 79 & 69 & 71 \\
\hline
\end{tabular}

The effect of noise removal on the retinal images is shown in Table II. Both $C B 1$ and $C B 2$ achieved better results compared to the results given in Table I. From the table it can be seen that the the use of $C B 2$ only results in better results than when using $C B 1$. $C B 2$ however, fails on a small subset of the images because, as noted above, the removal of the OD results in obscuration of drusen and consequently missclassifications result. As will be demonstrated (Table III), the combination of the two processes, resolves this problem.

Table III gives the results produced using the proposed approach. By comparing the results presented in Tables II and III it can be seen that the proposed two stage histogram based image classification process (using both $C B 1$ and $C B 2$ ) outperforms classification using $C B 2$ only, which in turn outperforms classification using $C B 1$ only. The combination of both case bases achieves an average accuracy improvement of $4 \%$ and $2 \%$ with respect to $C B 1$ and $C B 2$ respectively.
TABLE II

EXPERIMENTAL RESULTS GENERATED USING TCV: (I) $C B 1$, AND (II) $C B 2$

\begin{tabular}{|l||c|c||c|c||c|c|}
\hline \multicolumn{1}{|c||}{ TCV } & \multicolumn{2}{c||}{ Specificity (\%) } & \multicolumn{2}{c||}{ Sensitivity (\%) } & \multicolumn{2}{c|}{ Accuracy (\%) } \\
\cline { 2 - 7 } run & $C B 1$ & $C B 2$ & $C B 1$ & $C B 2$ & $C B 1$ & $C B 2$ \\
\hline 1 & 40 & 60 & 89 & 89 & 71 & 79 \\
2 & 67 & 67 & 75 & 88 & 71 & 79 \\
3 & 67 & 67 & 67 & 78 & 67 & 73 \\
4 & 67 & 67 & 89 & 89 & 80 & 80 \\
5 & 67 & 67 & 89 & 67 & 80 & 67 \\
6 & 50 & 50 & 100 & 89 & 80 & 73 \\
7 & 33 & 50 & 88 & 100 & 64 & 79 \\
8 & 60 & 60 & 67 & 67 & 64 & 64 \\
9 & 50 & 67 & 75 & 88 & 64 & 79 \\
10 & 67 & 67 & 75 & 63 & 71 & 65 \\
\hline Ave. & 57 & 62 & 81 & 81 & 71 & 73 \\
\hline
\end{tabular}

The improvement in average specificity is $8 \%$ and $3 \%$ with respect to $C B 1$ and $C B 2$, and $1 \%$ in the case of average sensitivity. The best average performance recorded is $82 \%$ sensitivity.

TABLE III

EXPERIMENTAL RESULTS GENERATED USING TCV FOR $C B 1$ AND $C B 2$ COMBINED

\begin{tabular}{|l||c|c|c|}
\hline TCV run & Specificity $(\%)$ & Sensitivity (\%) & Accuracy (\%) \\
\hline 1 & 60 & 89 & 79 \\
2 & 67 & 88 & 79 \\
3 & 67 & 78 & 73 \\
4 & 67 & 89 & 80 \\
5 & 83 & 67 & 73 \\
6 & 67 & 89 & 80 \\
7 & 50 & 88 & 71 \\
8 & 60 & 67 & 64 \\
9 & 67 & 88 & 79 \\
10 & 67 & 75 & 71 \\
\hline Average & 65 & 82 & 75 \\
\hline
\end{tabular}

A thorough analysis of the results found that OD removal effected only a small part of the histograms. Figure 5 compares a green channel histograms curve before OD removal (but with blood vessel pixels removed), and after OD removal. As can be seen from the figure the resulting modifications of the histogram curves is small. Most of the changes are located on the right side of the horizontal axis of the histogram. This is expected since most of the OD is constructed of the brightest pixels in the retinal image. However, the features of DTW that measures distance between a point in a time series curve to all points in the comparator time series curve to identify the shortest distance, makes the classifier sensitive to small changes in the generated curves (sufficient to give an increase in accuracy of $2 \%$ as shown in Table III). Consequently, a more distinctive pattern can be observed between classes (AMD and normal classes) that reflects the classification performance. It is also worth noting that all images that went to the second stage classification are those images where the OD was successfully segmented (i.e. there were no images where the OD could not be identified). 


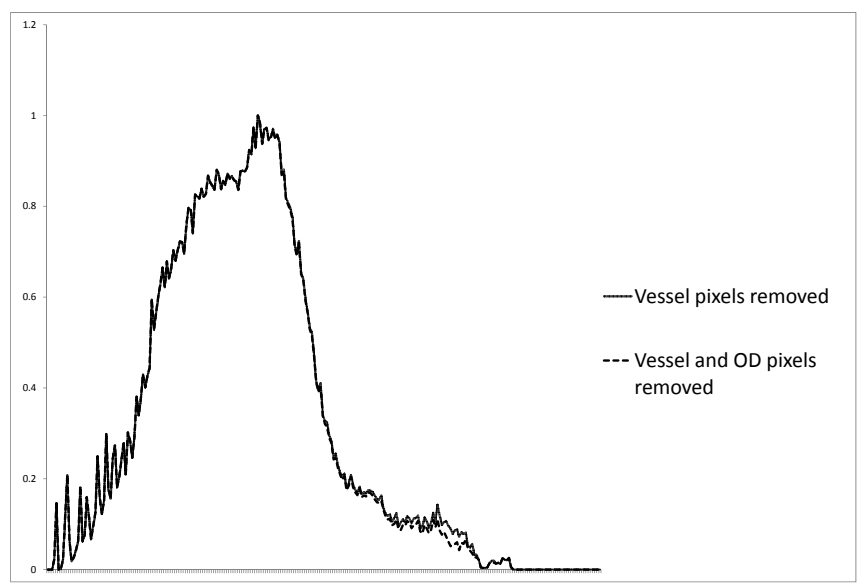

Fig. 5. Green channel histogram curves before and after OD removal

\section{SUMMARY AND CONCLUSIONS}

An approach to classify retinal images, in the context of AMD, using histograms and a DTW comparison technique has been proposed in this paper. Two content based histograms, green and saturation, were used to represent each image. Prior to histograms extraction, pre-processing was applied so as to enhance the images and remove unwanted noise (blood vessels, or blood vessels and the OD). The enhancement was directed at reducing both colour and illumination variation. A $2 D$ matched filter approach was applied to filter out retinal blood vessels. The main advantage of this technique was simplicity. OD removal was achieved using $2 D$ to two $1 D$ projection for OD localisation.

The idea of employing a two stage image classification process founded on two case bases has been demonstrated, at least in the case of the AMD application, to produced better classification results than one stage methods. In this paper, the first case base, $C B 1$, comprised histograms without retinal blood vessels information. The second case base $(C B 2)$ also excluded OD information. The best result achieved was sensitivity of $82 \%$. It is worth noted that in other work [6], the mean sensitivity achieved through graders observation on different set of retinal images was $86 \%$.

The authors intend to take the work forward by applying an image segmentation technique that will allow different parts of the retina images to be analised independently. The intention is to focus the classification process on the central area of the retinal image (the macula), the most likely location for drusen. The authors are also investigating ways to increase the size of the available data set.

\section{REFERENCES}

[1] S. Chaudhuri, S. Chatterjee, N. Katz, M. Nelson, and M. Goldbaum, "Detection of blood vessels in retinal images using two-dimensional matched filters," IEEE Transactions on Medical Imaging, vol. 8, no. 3, pp. 263-269, 1989.

[2] M. H. A. Hijazi, F. Coenen, and Y. Zheng, "A histogram approach for the screening of age-related macular degeneration," in Medical Image Understanding and Analysis 2009. BMVA, 2009, pp. 154-158.
[3] R. D. Jager, W. F. Mieler, and J. W. Mieler, "Age-related macular degeneration," The New England Journal of Medicine, vol. 358, no. 24, pp. 2606-2617, 2008.

[4] K. Rapantzikos, M. Zervakis, and K. Balas, "Detection and segmentation of drusen deposits on human retina: Potential in the diagnosis of age-related macular degeneration," Medical Image Analysis, vol. 7, pp. 95-108, 2003.

[5] C. Köse, U. Sevik, and O. Gençalioğlu, "Automatic segmentation of age-related macular degeneration in retinal fundus images," Computers in Biology and Medicine, vol. 38, pp. 611-619, 2008.

[6] S. Jain, S. Hamada, W. L. Membrey, and V. Chong, "Screening for age-related macular degeneration using nonstereo digital fundus photographs," Eye, vol. 20, pp. 471-475, 2006.

[7] Z. A. Aghbari, "Effective image mining by representing color histograms as time series," Journal of Advanced Computational Intelligence and Intelligent Informatics, vol. 13, no. 2, pp. 109-114, 2009.

[8] A. Conci and E. M. M. Castro, "Image mining by content," Expert System with Applications, vol. 23, pp. 377-383, 2002.

[9] P. G. Foschi, D. Kolippakkam, H. Liu, and A. Mandvikar, "Feature extraction for image mining," in International Workshop on Multimedia Information Systems, 2002, pp. 103-109.

[10] Y. Rubner, C. Tomasi, and L. J. Guibas, "The earth mover's distance as a metric for image retrieval," International Journal of Computer Vision, vol. 40, no. 2, pp. 99-121, 2000.

[11] R. Brunelli and O. Mich, "Histograms analysis for image retrieval," Pattern Recognition Letters, vol. 34, pp. 1625-1637, 2001.

[12] A. A.-H. A.-R. Youssif, A. Z. Ghalwash, and A. A. S. A.-R. Ghoneim, "Optic disc detection from normalized digital fundus images by means of a vessel's direction matced filter," IEEE Transactions on Medical Imaging, vol. 27, no. 1, pp. 11-18, 2008.

[13] J. Kolodner, Case-based reasoning. Morgan Kaufmann, 1993.

[14] C. S. Myers and L. R. Rabiner, "A comparative study of several dynamic time-warping algorithms for connected word recognition," The Bell System Technical Journal, vol. 60, no. 7, pp. 1389-1409, 1981.

[15] J. D. Cryer and K.-S. Chan, Time Series Analysis: With Applications in $R$. Springer, 2008.

[16] R. C. Gonzalez and R. E. Woods, Digital image processing, R. C. Gonzalez and R. E. Woods, Eds. Pearson Prentice Hall, 2008.

[17] M. Foracchia, E. Grisan, and A. Ruggeri, "Luminosity and contrast normalization in retinal images," Medical Image Analysis, vol. 9, pp. 179-190, 2005.

[18] A. Osareh, "Automated identification of diabetic retinal exudates and the optic disc," Ph.D. dissertation, University of Bristol, UK, 2004.

[19] K. Zuiderveld, Contrast limited adaptive histogram equalization, ser Academic Press Graphics Gems Series. Academic Press Professional, Inc., 1994, pp. 474-485.

[20] N. Patton, T. M. Aslam, and T. MacGillivray, "Retinal image analysis: Concepts, applications and potential," Progress in Retinal and Eye Research, vol. 25, pp. 99-127, 2006.

[21] N. Otsu, "A threshold selection method from gray level histograms," IEEE Transactions on Systems, Mans and Cybernetics, vol. SMC-9, no. 1 , pp. 62-66, 1979.

[22] A. E. Mahfouz and A. S. Fahmy, "Ultrafast localisation of the optic disc using dimensionality reduction of the search space," in proceedings of Medical Image Computing and Computer-Assisted Intervention, 2009, pp. 985-992.

[23] D. J. Berndt and J. Clifford, "Using dynamic time warping to find patterns in time series," in AAAI Workshop on Knowledge Discovery in Databases, 1994, pp. 229-248.

[24] E. J. Keogh and M. J. Pazzani, "Derivative dynamic time warping," in First SIAM International Conference on Data Mining, 2001. 